\title{
Inhibition of matrix metalloproteinases in Siberian hamsters impedes photostimulated recrudescence of ovaries
}

\author{
Julie Whited, Asha Shahed, Carling F McMichael and Kelly A Young \\ Reproductive Biology Group, Department of Biological Sciences, California State University Long Beach, Long Beach, \\ California 90840-3702, USA \\ Correspondence should be addressed to K A Young; Email: kayoung@csulb.edu
}

J Whited is now at Department of Pathology and Clinical Medicine, David Geffen School of Medicine at UCLA, 10833 Le Conte Avenue, Los Angeles, California 90095-1732, USA

\begin{abstract}
Exposure of Siberian hamsters to short photoperiod for 14 weeks induces ovarian regression. Subsequent transfer to long photoperiod restores ovarian function, and 2 weeks of photostimulation increases plasma estradiol $\left(E_{2}\right)$, antral follicles, and corpora lutea $(C L)$. Because tissue remodeling involved with photostimulated ovarian recrudescence is associated with differential expression of matrix metalloproteinases (MMPs), we hypothesized that inhibiting MMP activity using a broad-spectrum in vivo MMP inhibitor, GM6001, would curtail recrudescence. One group of hamsters was placed in long days (LD; 16 h light:8 h darkness) for 16 weeks. Another group was placed in inhibitory short days (SD; $8 \mathrm{~h}$ light:16 $\mathrm{h}$ darkness) for 14 weeks. A third group was placed in SD for 14 weeks and transferred to LD for 2 weeks to stimulate recrudescence. During weeks 14-16, animals were either not treated or treated daily with i.p. injections of GM6001 (20 mg/kg) or vehicle (DMSO). GM6001 reduced gelatinase activity and decreased immunohistochemical staining for MMP1, MMP2, and MMP3 compared with vehicle. No differences between controls, vehicle, or GM6001 treatment were observed among LD animals, despite a trend toward reduction in $C L$ and $E_{2}$ with $G M 6001$. Although $S D$ reduced ovarian function, photostimulation of transferred controls increased uterine mass, plasma $E_{2}$, appearance of antral follicles, and CL. With GM6001 treatment, photostimulation failed to increase uterine mass, plasma $E_{2}$, antral follicles, or CL. These data show, for the first time, that in vivo GM6001 administration inhibits MMP activity in hamster ovaries during photostimulation, and indicate that this inhibition may impede photostimulated recrudescence of ovaries. This study suggests an intriguing link between MMP activity and return to ovarian function during photostimulated recrudescence.

Reproduction (2010) $\mathbf{1 4 0} 875-883$
\end{abstract}

\section{Introduction}

To maximize survival of parent and offspring, many temperate mammals limit reproduction to times when resources are more plentiful. Seasonal limitation of reproduction can be regulated by environmental cues such as food availability, ambient temperature, and photoperiod. Siberian hamsters (Phodopus sungorus) experience a physiological loss and return of reproductive function mediated by photoperiod exposure that can be recreated in the laboratory. Exposure to short photoperiod causes extended periods of melatonin release, triggering a cascade of hormonal changes that suppress activity of the hypothalamic-pituitary-gonadal (HPG) axis, reducing synthesis and secretion of pituitary gonadotropins, and eventually leading to regression of the ovaries and inhibition of reproductive function (Schlatt et al. 1993, Moffatt-Blue et al. 2006). Exposure to $12-14$ weeks in short $(8 \mathrm{~h}$ light:16 h darkness per day; short day (SD)) photoperiod is sufficient to induce regression of the ovaries, anovulation, and decreased estradiol $\left(\mathrm{E}_{2}\right)$ production (Schlatt et al. 1993, Moffatt-Blue et al. 2006, Salverson et al. 2008). Reproductively, regressed hamsters are characterized by the absence of corpora lutea $(\mathrm{CL})$ in the ovaries, decreases in uterine mass and plasma concentration of $\mathrm{E}_{2}$, and the presence of eosiniphilic terminal atretic follicles, structures typically found only in regressed ovaries (van den Hurk et al. 2002, Moffatt-Blue et al. 2006, Kabithe \& Place 2008). Once transferred to long day (LD) photoperiods of $16 \mathrm{~h}$ light: $8 \mathrm{~h}$ darkness per $24 \mathrm{~h}$ period, female hamsters will rapidly regain reproductive function (Salverson et al. 2008, Shahed \& Young 2008). LD photostimulation increases the number of $\mathrm{CL}$ within 1 week, decreases the presence of terminal atretic follicles by 2 weeks, and restores plasma $\mathrm{E}_{2}$ concentrations by 4 weeks (Salverson et al. 2008). These changes require significant ovarian remodeling at the cellular level. 
In the breeding season, ovaries undergo continuous tissue remodeling during follicular growth, maturation, and ovulation. A family of proteins known as the matrix metalloproteinases (MMPs) play an important role in this process by clearing the way for new growth by cleaving ovarian tissue components, releasing growth factors, and contributing to the degradation of the extracellular matrix (Curry \& Osteen 2003, Smith et al. 2005). Although the role of these proteases in ovulation and development of CL is not fully defined (Liu et al. 2006, Wahlberg et al. 2007), MMPs and their endogenous inhibitors (tissue inhibitors of MMPs) are differentially expressed in the cycling ovary and uterus (Fata et al. 2000, Curry \& Osteen 2001, Vrooman \& Young 2010), and are implicated in regulation of estrus and menstrual cycles.

Because differential expression of MMPs also occurs during ovarian recrudescence (Salverson et al. 2008), we hypothesized that an inhibition of MMP activity would impede LD-stimulated ovarian return to function. MMPs share a basic structural organization and are dependent on zinc for their activity (Bauvois 2001). GM6001, also known as galardin, ilomastat, or $\mathrm{N}-((2 \mathrm{R})$-2-(hydroxamidocarbonylmethyl)-4-methylpentanoyl)-L-tryptophan methylamide, is a peptide hydroxamate broad-spectrum inhibitor of MMPs, which works in vivo by chelating the active site zinc atom (Grobelny et al. 1992; Millipore BIOMOL Research Laboratories 2009, GM6001 product data sheet). We specifically hypothesized that in vivo administration of GM6001 would curtail the effects of photostimulated recrudescence during 2 weeks of LD stimulation following chronic SD exposure.

\section{Results}

\section{Reproductive organ masses}

Ovarian mass did not differ between LD-exposed no-treatment controls (LD) and the LD vehicle (LDV), LD GM6001 (LDG), post-transfer, and post-transfer vehicle (PTV) groups (Fig. 1). In contrast, ovarian mass was reduced 1.8-2.0-fold with SD exposure and following the GM6001 treatment during post-transfer exposure to LD photoperiod (PTG) as compared with LD, PT, and PTV controls $(P<0.05$; Fig. $1 \mathrm{~A})$. Uterine mass remained unchanged across the $\mathrm{LD}, \mathrm{LDV}, \mathrm{LDG}, \mathrm{PT}$, and PTV groups $(P>0.05$; Fig. 1B), however, decreased in the SD (3.0-fold) and PTG groups (2.6-fold) as compared with LD controls, and declined 2.5- and 2.2-fold as compared with the PTV females respectively ( $P<0.05$; Fig. 1B).

\section{Follicle counts}

Ovarian histology was evaluated and follicles were quantified for the number of preantral, antral, $\mathrm{CL}$, atretic, and terminal atretic follicles (Fig. 2). High numbers of $\mathrm{CL}$, antral, and preantral follicles were noted in $\mathrm{LD}$
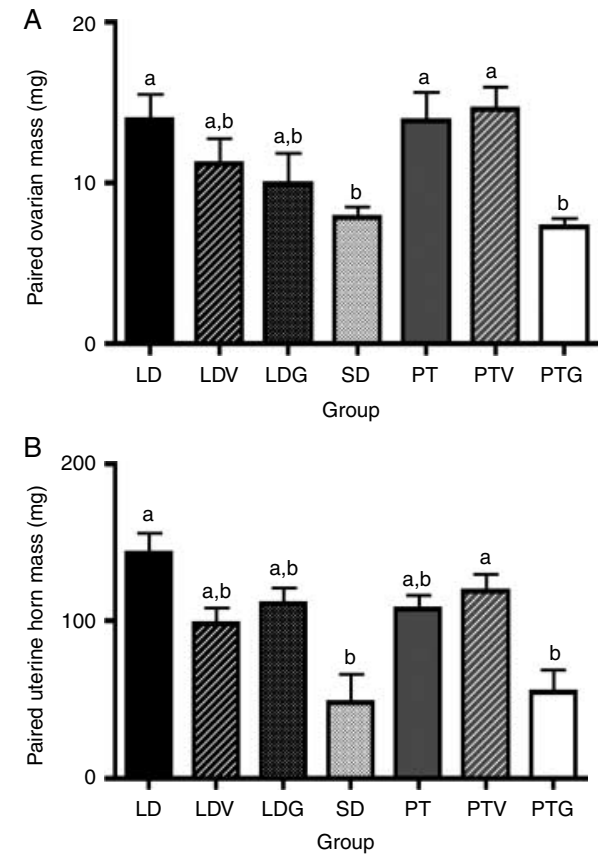

Figure 1 Paired ovary mass (A) and paired uterine horn mass (B). Mean \pm S.E.M. (A) ovarian mass $(\mathrm{g})$ and $(\mathrm{B})$ uterine mass $(\mathrm{g})$ in Siberian hamsters exposed to long day (LD, $n=9$; LDV, $n=9$; LDG, $n=9$ ); animals transferred from short day (SD) to LD photoperiod (PT, $n=4$; PTV, $n=7$ ); animals exposed to SD photoperiod alone (SD, $n=4)$ and animals treated with GM6001 during exposure to LD photoperiod (PTG, $n=9$ ). Groups with different letters are significantly different $(P<0.05)$.

controls, as expected (Fig. 2A). Active ovaries with preantal and antral follicles were also observed in the LDG females treated with GM6001 (Fig. 2B). Exposure to $\mathrm{SD}$ conditions for 14 weeks reduced the number of $\mathrm{CL}$ and antral follicles, and increased the numbers of eosiniphilic terminal atretic follicles as compared with LD females (Fig. 2C). Subsequent transfer to LD conditions for 2 weeks (PT) increased CL and antral follicles, and eliminated terminal atretic follicles (Fig. 2D). In contrast, post-transfer females treated with GM6001 (PTG) showed low numbers of CL and antral follicles, and high numbers of terminal atretic follicles (Fig. 2E). Vehicle administration had no apparent effect on LD or PT females; no general differences were observed (data not shown, but quantified below).

When quantified, follicle counts did not vary significantly between the LD, LDV, LDG, PT, and PTV groups for any follicle type (Fig. 3). The number of preantral follicles did not differ significantly between any groups (Fig. 3A). Antral follicle numbers were highest in LD, LDV, LDG, PT, and PTV, and there were no differences across these groups $(P>0.05$; Fig. $3 \mathrm{~B})$. In contrast, no antral follicles were noted in any SD or PTG female, a 2.5-fold decrease as compared with LD, LDG, and PT ovaries ( $P<0.05$; Fig. 3B). LD and LDV females had high number of $\mathrm{CL}$; treatment with GM6001 did not 
A

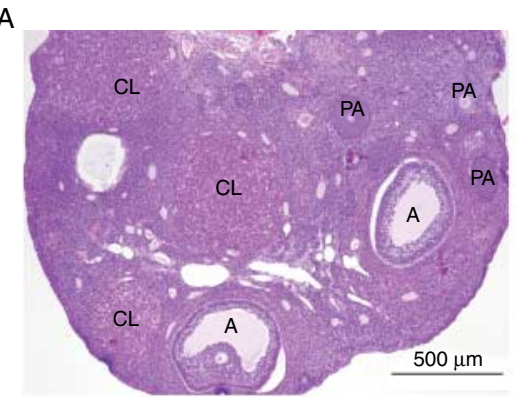

C
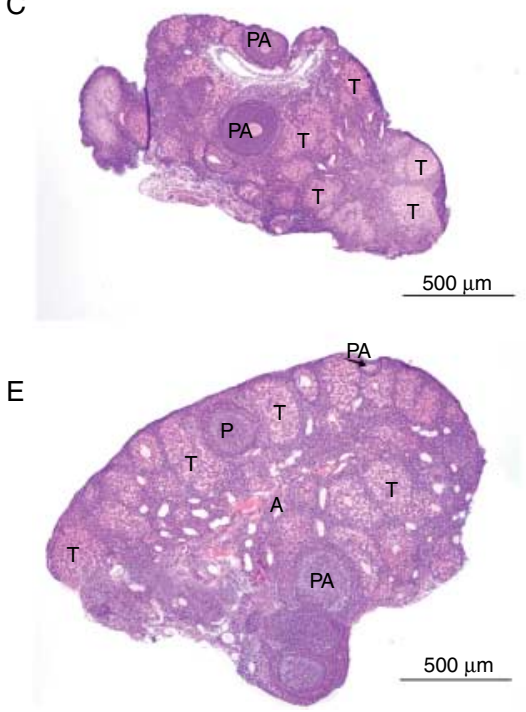

B
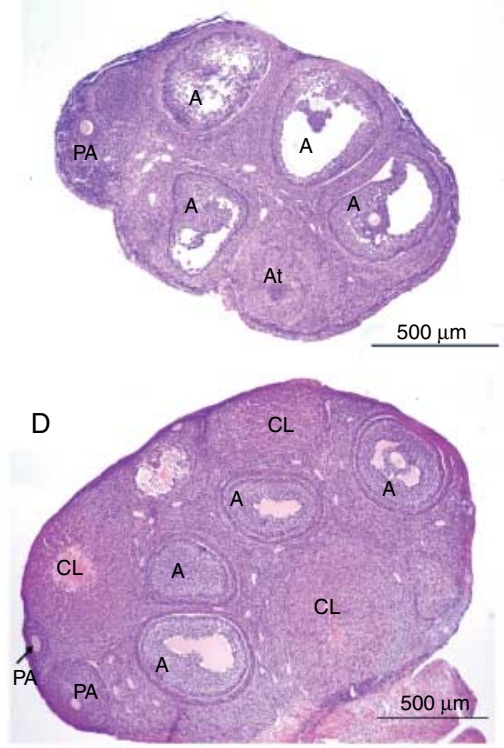

Figure 2 Representative cross sections of ovaries from Siberian hamsters stained with hematoxylin and eosin. (A) Long day (LD) control. (B) LD with GM6001 treatment (LDG). (C) Short day (SD).

(D) Post-transfer with vehicle treatment (PTV). (E) Post-transfer with GM6001 treatment (PTG). $\mathrm{CL}$, corpus luteum; $\mathrm{P}$, preantral follicles; $\mathrm{A}$, antral follicles; $\mathrm{T}$, terminal atretic follicles, At, atretic follicles; $n$ per group the same as listed in Fig. 1 .

significantly decrease CL numbers in the LDG group, although a declining trend was apparent $(P>0.05$; Fig. 3C). CL were not present in SD females, a significant decrease as compared with the LD, LDV, LDG, PT, and PTV groups $(P<0.05$; Fig. 3C). Transfer to LD photostimulation restored ovulatory events in PT and PTV females; these females had $\mathrm{CL}$ numbers no different from those observed in LD controls $(P>0.05$; Fig. 3C). Interestingly, CL numbers were reduced 11 -fold in PTG females compared with LD ovaries, and 10-fold compared with PTV $(P<0.05$; Fig. $3 \mathrm{C})$. When quantified, no differences were noted in standard atretic follicles $(P>0.05$, data not shown); however, terminal atretic follicles characteristic of SD regressed hamster ovaries were noted only in the SD and PTG groups, with significant differences observed in SD and PTG females as compared with all other groups ( $P<0.05$; Fig. 3D).

\section{Blood plasma $E_{2}$ concentration}

Concentrations of plasma $E_{2}$ for LD animals were within typical values for reproductively cycling females of this species (Moffatt-Blue et al. 2006, Salverson et al. 2008). $E_{2}$ values did not differ with vehicle or GM6001 administration in LD females, despite a downward trend in the LDG group ( $P>0.05$; Fig. 4). Exposure to inhibitory photoperiod reduced plasma $E_{2}$ values 11.5 -fold in the SD group as compared with LD levels $\left(P<0.05\right.$; Fig. 4). Plasma $\mathrm{E}_{2}$ concentrations in $\mathrm{PT}$ and PTV females were restored to levels no different from those observed in LD females: PT and PTV $E_{2}$ concentrations did not differ as compared with all other groups ( $P>0.05$; Fig. 4). In contrast, treatment with GM6001 prevented the increase in $E_{2}$ concentrations observed in the PT group; values in PTG females were 1.5-2.0-fold lower as compared with PT and PTV animals, and 2.5-fold lower $(P<0.05)$ as compared with the LD and LDV groups (Fig. 4).

\section{MMP expression and activity}

Treatment with GM6001 significantly reduced the activity of active MMP2 (Fig. 5B) and MMP9 (Fig. 5C) in the uterine tissue as compared with the PTV group $(P<0.05)$. There was no difference in MMP2 or MMP9 activity between the LDV and PTV groups $(P>0.05)$. As an additional control to demonstrate that GM6001 reduces MMP activity, immunostaining levels were quantified for pro/active MMP1, MMP2, and MMP3 across the PTV and PTG groups (Fig. 6). Interstitial collagenase (MMP1) staining levels were 2.8 -fold lower in PTG as compared with PTV females $(P<0.05$; Fig. 6A 

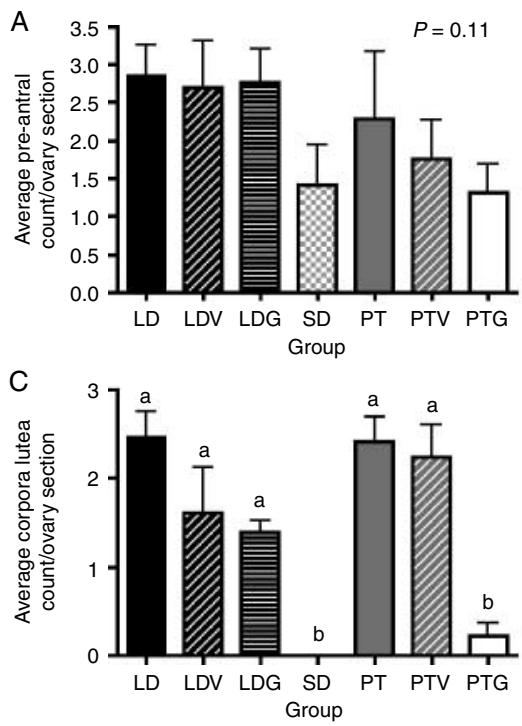
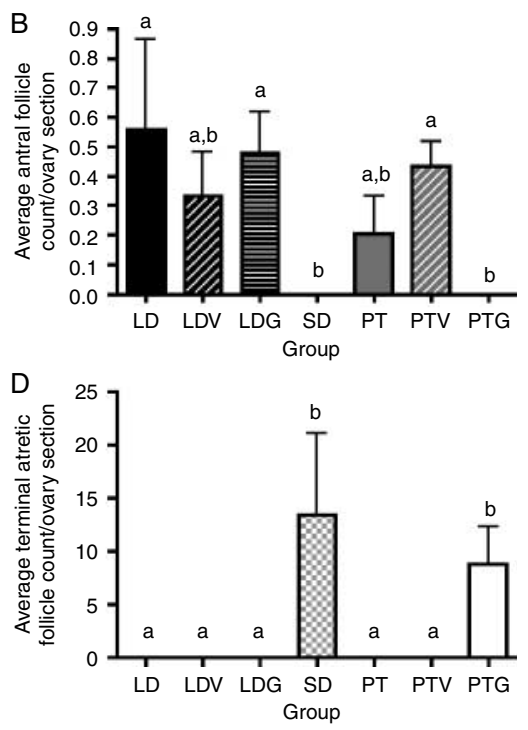

Figure 3 Mean \pm S.E.M. number of (A) preantral follicles, (B) antral follicles, (C) corpora lutea, and (D) terminal atretic follicles per ovarian cross section in Siberian hamsters exposed to long day (LD), short day (SD), or post-transfer (PT) from SD to LD photoperiod with no treatment (LD, SD, and PT), or with treatment with vehicle (LDV and PTV) or GM6001 (LDG and PTG). Groups with different letters are significantly different $(P<0.05) ; n$ per group the same as listed in Fig. 1. and C). MMP2 immunostaining was also reduced in the PTG group as compared with the PTV group $(P<0.05$; Fig. 6D-F), and similarly, MMP3 immunostaining declined threefold in the PTG as compared with the PTV group $(P<0.05$; Fig. 6G-I). No staining was noted in controls processed in parallel without primary antibody (Fig. 6). Finally, because GM6001 affects MMPs at the activity level, mRNA expression of MMP9 and MMP2 was examined in the ovarian tissue using real-time PCR and species-specific primers to ensure that a global reduction in transcription had not occurred. The expression of MMP2 and MMP9 mRNA remained unchanged between the PTV controls and GM6001-treated (PTG) groups, as expected $(P>0.05$, data not shown).

\section{Discussion}

This study is the first to demonstrate an alteration of normal ovarian function during photoperiod-mediated recrudescence as a result of reducing MMP activity. While transfer from inhibitory short photoperiods into stimulatory long photoperiods restored reproductive function via increased ovarian and uterine mass, follicular development, ovulation and $E_{2}$ production, treatment of regressed females with MMP inhibitor GM6001 prevented these increases during photostimulation. Indeed, regressed females treated with MMP inhibitor maintained an SD phenotype post-transfer to LD: reduced follicle development, ovulation, $\mathrm{E}_{2}$ concentrations, ovarian and uterine mass, and the presence of terminal atretic follicles are the characteristic of regressed ovaries. Because an inhibition of MMPs using GM6001 impedes ovarian recrudescence at 2 weeks of treatment, our study demonstrates a potential role for MMPs during photostimulated return of ovarian function in Siberian hamsters. To our knowledge, these data provide the first evidence that ovarian function during photostimulated recrudescence can be impeded via inhibition of a protease class at the level of the reproductive organs (ovary, uterus).

MMP inhibition was demonstrated using gelatin zymography and immunohistochemistry. In addition to ovarian tissue, uterine tissue was used for these control experiments because 1) the uterus is a target organ of the ovaries and is an excellent measure of reproductive function, and 2) ovarian tissue is limited (3-5 mg tissue for the SD and PTG groups). GM6001 works at the protein level, inhibiting MMP activity via chelation of the active site zinc atom (Grobelny et al. 1992); therefore, zymography was critical to show a reduction in actual MMP activity, and active forms of both MMP2 and MMP9 were significantly reduced following GM6001 treatment (Fig. 5). Similarly, all primary antibodies used for immunohistochemical staining recognized both pro-form and active form of MMPs; GM6001 inhibits

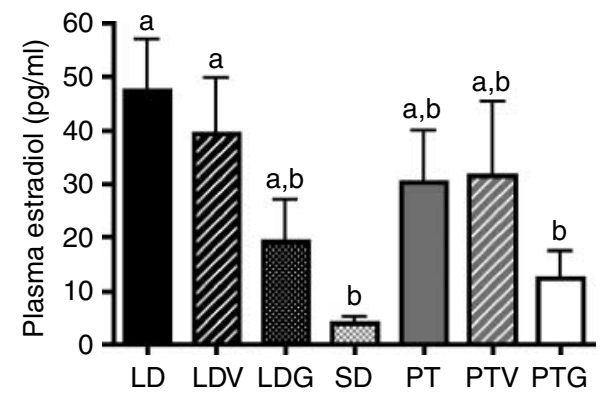

Figure 4 Plasma estradiol concentrations $(\mathrm{pg} / \mathrm{ml})$ in recrudescing Siberian hamsters. Mean \pm S.E.M. plasma estradiol concentrations $(\mathrm{pg} / \mathrm{ml})$ in Siberian hamsters exposed to long day (LD), short day (SD), or post-transfer (PT) from SD to LD photoperiod with no treatment (LD, SD, and PT), or with treatment with vehicle (LDV and PTV) or GM6001 (LDG and PTG). Groups with different letters are significantly different $(P<0.05)$; $n$ per group the same as listed in Fig. 1 . 


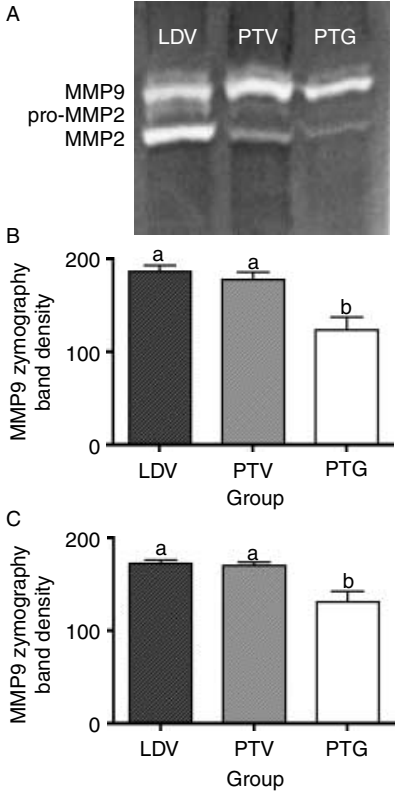

Figure 5 Protease activity of MMP2 and MMP9 in uterine protein extracts in recrudescing Siberian hamsters. (A) Gelatin zymogram. Active MMP9 (80-82 kDa), pro-MMP2 (72 kDa), and active MMP2 (60 kDa) bands are labeled. (B) Mean \pm s.E.M. active MMP2 and (C) MMP9 activity in Siberian hamsters exposed to LD with vehicle treatment (LDV), post-transfer with vehicle treatment (PTV), and posttransfer with GM6001 treatment (PTG). Groups with different letters are significantly different $(P<0.05) ; n$ per group the same as listed in Fig. 1.

activation of MMPs and thus a potential reduction of MMP immunostaining, rather than an absence, was predicted. Staining for collagenase (MMP1), gelatinase (MMP2), and stromelysin (MMP3) was significantly reduced with use of the GM6001 inhibitor (Fig. 6). While it is not known whether the reduction in immunostaining reflects a decline in the pro-form, active form, or both, GM6001 administration did induce a decrease in these MMPs. As an additional demonstration of efficacy of GM6001 at the level of activity, real-time PCR results showed that the expression of MMP9 and MMP2 mRNA remained unchanged between the vehicle and GM6001-treated groups. This was as expected, since GM6001 inhibits MMP activity and not mRNA expression. The inhibition of ovarian function observed in PTG females did not appear to result from systemic influences of the inhibitor on overall health of the hamsters. Compared with related studies using GM6001 in vivo, the $20 \mathrm{mg} / \mathrm{kg}$ body weight dose used in this study was in the mid to low range (Liu et al. 2006). Animals treated with GM6001 in this study showed no visible signs of distress during the 2-week treatment period, regardless of being in the LDG or PTG groups.

Administration of GM6001 in the control (cycling) animals did not affect ovarian or uterine mass, though masses did not differ significantly in this group from either PTG or SD females (Fig. 1). Similarly, GM6001 administration to LD females did not affect preantral or antral follicle counts as compared with LD or LDV controls, although it was noted that several antral follicles were counted as atretic in the GM6001-treated group, as opposed to the more typically observed secondary follicle undergoing atresia (Fig. 2). While CL numbers in the LDG group did not significantly decline as compared with LD females, CL number appeared to trend downward, with a 56\% drop as compared with LD controls, which could reflect a possible decline in ovulation or $\mathrm{CL}$ function when MMPs are inhibited in LD control hamsters (post hoc ANOVA comparing only LD, LDV, LDG CL numbers, $P=0.06$ ). Plasma $E_{2}$ concentrations also showed a nonsignificant decline in LDG females, with levels $40 \%$ lower than LD controls. A minor $(20 \%)$, but significant, decrease in ovulation efficiency does occur in pregnant mares serum gonadotrophin/human chorionic gonadotrophin-stimulated immature C57BL/6 mice administered GM6001 at a dose of $100 \mathrm{mg} / \mathrm{kg}$ (Liu et al. 2006), although no differences were observed in number of $\mathrm{CL}$ formed (Wahlberg et al. 2007). These studies used a higher dose than the $20 \mathrm{mg} / \mathrm{kg}$ used in this study, and concluded that MMPs may not be obligatory for ovulation and $\mathrm{CL}$ development in mice (Liu et al. 2006, Wahlberg et al. 2007). However, because MMPs are expressed and differentially regulated in the ovary during follicle development, ovulation, and $\mathrm{CL}$ formation/degradation (Curry \& Osteen 2003, Vrooman \& Young 2010), they may play a role in normal ovarian function that could be potentially teased out with a dedicated dose-response study using GM6001 (or other more specific inhibitors, such as gelatinase inhibitor SB3T) and LD control females. It is also likely that the MMPs inhibited with GM6001 are just one part of a large array of proteases and related proteins that mediate the tissue remodeling of folliculogenesis. At the low dose administered, however, no significant changes were noted between LD females in the control, vehicle, and GM6001 groups. In contrast, inhibition of MMPs in regressed females exposed to LD photostimulation retarded resumption of ovarian function, including mass, folliculogenesis, ovulation, and $E_{2}$ production. After transfer to LD photoperiod for 2 weeks, hamsters treated with GM60001 inhibitor had ovaries that resembled regressed SD ovaries, suggesting that MMPs may play a more prominent role in the tissue remodeling required for recrudescence, and that regulation of this remodeling may differ from that in normal ovarian cycling. Because MMPs play key roles in the rapid growth associated with tumorigenesis (Kessenbrock et al. 2010), their function may be more prominent during photostimulated ovarian restoration. Alternatively, regressed ovaries may be more sensitive to MMP inhibition, and differential expression of MMPs noted during photostimulated recrudescence (Salverson et al. 2008) may simply reflect a return to normal low levels of MMP action. 
A
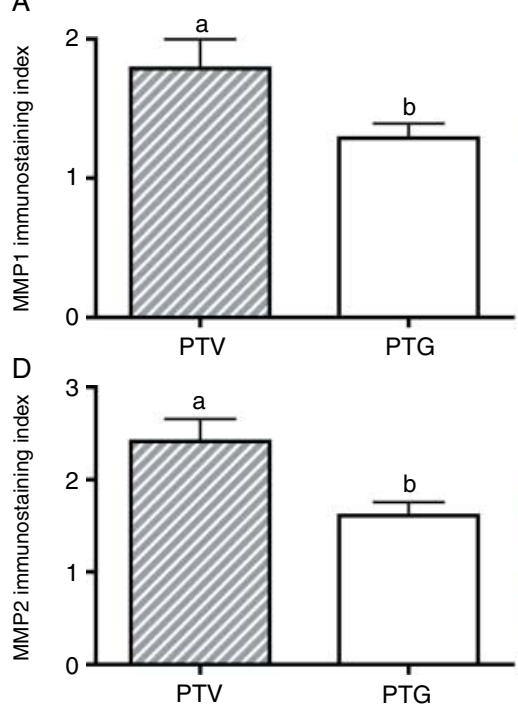

G

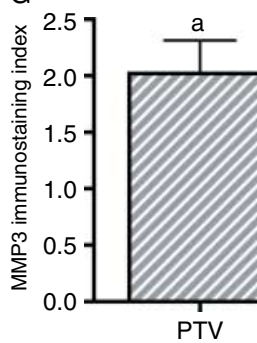

B

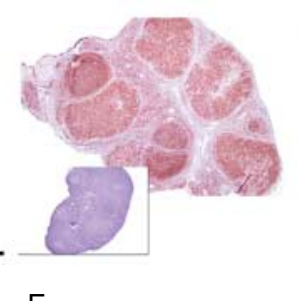

$\mathrm{E}$

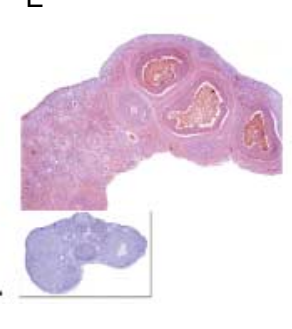

$\mathrm{H}$

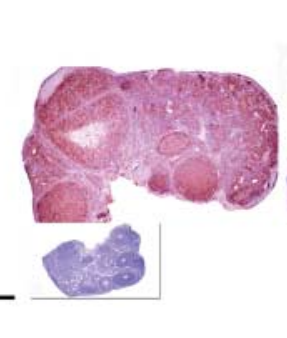

C

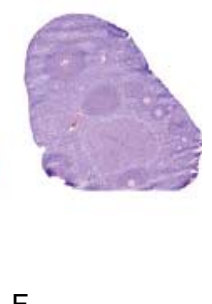

$\mathrm{F}$

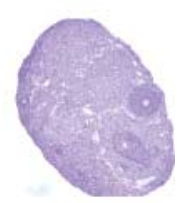

I

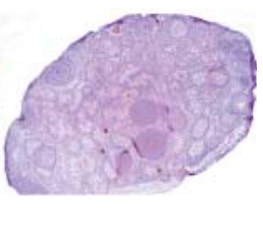

Figure 6 Extent and intensity of immunostaining for MMPs. Mean \pm S.E.M. immunostaining index levels (scores of 0-3) indicating extent and intensity of $\mathrm{red} /$ pink-stained cells in Siberian hamsters post-transfer from short day to long day photoperiod with vehicle (PTV) or GM6001 (PTG) treatment. (A) MMP1 staining index, typical (B) high and (C) low MMP1 immunostaining, (D) MMP2 staining index, typical (E) high and (F) low MMP2 immunostaining, (G) MMP1 staining index, typical $(\mathrm{H})$ high and (I) low MMP1 immunostaining. Insets depict negative controls processed without primary antibody; $n=4-9$ /group. Groups with different letters are significantly different $(P<0.05)$.
Because natural intra-ovarian MMP activity is regulated at the level of transcription, translation, secretion, and via activators and inhibitors, MMP involvement in ovarian recrudescence includes more than just the MMPs inhibited by GM6001. Gonadotropins and sex steroid hormones can regulate MMP activity (Jo et al. 2004, Young \& Stouffer 2004), and both $\mathrm{SD}$ exposure and recrudescence alter $\mathrm{FSH}$ and plasma $\mathrm{E}_{2}$ in Siberian hamsters (Schlatt et al. 1993, Moffatt-Blue et al. 2006). In turn, MMP activity can influence steroid synthesis in gonadal tissue (e.g. Nothnick 2003, Evaul \& Hammes 2008) and, in this study, inhibition of MMP activity influenced $E_{2}$ production in the recrudescing ovary. While the decrease in $\mathrm{E}_{2}$ production is concomitant with GM6001 administration, it is not known whether this decline is a result of the direct influence of MMP activity on steroidogenesis, via a possible mechanism of growth factor release, or whether the decline results simply by impeding granulosa cell proliferation and follicle development in general. MMPs can also regulate and activate each other, in particular, the activation of pro-MMP2 via membranebound MT-MMPs (e.g. Butler et al. 1997, Shofuda et al. 1997). Ovarian MT-MMP1 (MMP14) peaks during weeks 1-2 of recrudescence (Salverson et al. 2008), and GM6001 inhibition of this branch of the MMP family may have multiple ramifications at the tissue level.
GM6001 has also been reported to inhibit some proteases in the ADAMs family as well as tumour necrosis factor $\alpha$ in the neural tissue (Galardy et al. 1994, Leib et al. 2000); these additional factors could also impact recrudescence at the level of the ovary. Finally, the transmembrane glycoprotein basinogen stimulates MMP activity and is expressed at high levels in the periovulatory period and during luteinization (Sun \& Hemler 2001, Kanekura et al. 2002). Owing to the influence MMPs have on recrudescence, basinogen and other MMP inducers may therefore be critical in regulating recrudescence in seasonal breeders. Examining these and other MMP regulators, and how they are controlled by the central HPG axis is critical to understand photostimulated return to ovarian function.

Taken together, the results of this study support the hypothesis that MMPaction is needed for timely return to ovarian function. This study is the first to inhibit recrudescence at the level of the ovary, and the first to show that MMP activity is needed for photostimulated restoration of ovarian function. Administration of the MMP inhibitor GM6001 to regressed females effectively reduces follicle development, ovulation, and $\mathrm{E}_{2}$ plasma concentration, whereas administration of vehicle alone allows return to function that is indistinguishable from recrudescing females receiving no treatment. Further studies to examine the substrates of these 
proteases, to establish MMP effects on specific indices of recrudescence such as angiogenesis, granulosa cell proliferation, and steroidogenesis, and use of more select inhibitors to specific MMPs are warranted to allow a better understanding of how nonfunctional ovarian tissue can return to a fully functional state following photostimulation.

\section{Materials and Methods}

\section{Animals}

Adult Siberian hamsters (P. sungorus) were obtained from the colony of Dr Katherine Wynne-Edwards, Queens University (Kingston, ON, Canada). Experiments were carried out at California State University Long Beach (CSULB) in AALAC approved facilities and were conducted in compliance with CSULB and NRC guidelines for the use of laboratory animals, and our approved IACUC protocol. Hamsters were given tap water and food (mixture of Lab Rodent Diet 5001 and Mazuri Hamster \& Gerbil Diet, Purina, Brentwood, MO, USA) ad libitum for the duration of the experiment and were housed individually in clear polypropylene cages. Female $(n=51)$ hamsters were acclimated to an LD (16L:8D) photoperiod for 3 weeks in a room with temperature maintained at $20 \pm 2{ }^{\circ} \mathrm{C}$. To ensure that the females' estrous cycle continues, male hamsters $(n=6)$ were placed among the females for the duration of the study. One arbitrarily assigned group of female hamsters $(n=24)$ was then exposed to an SD (8L:16D) photoperiod for 14 weeks in order to ensure full ovarian regression (after Salverson et al. 2008), while remaining hamsters stayed in LD photoperiod as controls. At the end of 14 weeks, tissue was collected from four female hamsters from the LD and four females from the SD groups. All remaining SD females $(n=20)$ were transferred back to LD conditions for 2 weeks in order to stimulate recrudescence of ovarian structures, and the LD females remained in long photoperiod (Table 1).

\section{Treatment with MMP inhibitor}

GM6001 (Millipore, Billerica, MA, USA) was dissolved in DMSO for a final concentration of $40 \mathrm{mg} / \mathrm{ml}$. During the 2 weeks following transfer to LD conditions, an experimental group of females (post-transfer week 2 with GM6001 treatment; PTG; $n=9$ ) was treated daily with i.p. injections of GM6001 (20 mg/kg body weight). Controls included an LD GM6001 group receiving the same dose (LDG; $n=9$ ), and both the LDV $(n=9)$ and PTV $(n=7)$ groups that were injected with an equal volume of DMSO vehicle. An additional set of control groups was not given injections (LD, $n=9$ total collected at 14 and 16 weeks and PT, $n=4$; Table 1 ). These selected time points were intended to represent breeding condition (LD), nonbreeding condition (SD), and early recrudescence (PT) respectively (Table 1). In order to minimize variations in plasma hormone levels and ovarian morphology due to differences in estrous cycle, soiled male bedding was added to the females' cages on the 12th day post-transfer to help synchronize estrous cycles prior to all tissue collection (Dodge et al. 2002, Moffatt-Blue et al. 2006, Salverson et al. 2008).

\section{Tissue collection}

On the day of tissue collection, female hamsters were weighed and blood samples were taken retro-orbitally with heparinized capillary tubes following a cocktail of ketamine $(20 \mathrm{mg} / \mathrm{kg})$ and xylazine $(200 \mathrm{mg} / \mathrm{kg})$ administered i.p. Each hamster was then euthanized via cervical dislocation and reproductive organs were immediately removed, dissected, and weighed. One ovary was fixed in $10 \%$ neutral buffered formalin for 7 days, and then transferred to $70 \%$ ethanol. The contralateral ovary was flash frozen in liquid nitrogen.

\section{$E_{2} R I A$}

Collected plasma was separated by centrifugation $(1500 \boldsymbol{g}$ for $5 \mathrm{~min}$ ) and stored at $-80^{\circ} \mathrm{C}$, until used in the $\mathrm{E}_{2}$ RIA with Ultra-Sensitive $E_{2}$ RIA ${ }^{125}$ I double antibody kits (Diagnostic Systems Laboratories, Inc., Webster, TX, USA). Samples were assayed in duplicate and their radioactivity was measured using a gamma counter. A standard curve was generated using a four-parameter logistic curve function (Sigma Plot software, SPSS, Inc., Chicago, IL, USA) and the final hormone concentrations were calculated using the Sigma Plot standard curve analysis function. Assay standards and controls were within the normal limits (Moffatt-Blue et al. 2006), with a $2.2 \mathrm{pg} / \mathrm{ml}$ lower limit of detection.

\section{Ovarian histology and follicle counts}

Fixed tissues were dehydrated in a graded series of ethanol solutions and xylenes, and embedded in paraffin wax. Serial paraffin sections of $6 \mu \mathrm{m}$ thickness were collected from every

Table 1 Experimental design for photoperiod exposure and GM6001 in vivo treatment of Phodopus sungorus.

\begin{tabular}{llll}
\hline Group & Photoperiod regime & Treatment & Purpose \\
\hline SD $(n=4)$ & 14-weeks SD & None & Control for regressed ovaries \\
LD $(n=9)$ & 16-weeks LD & None & Control for fully functional ovaries \\
LDV $(n=9)$ & 16 -weeks LD & DMSO vehicle injection & Control for inhibitor administration \\
LDG $(n=9)$ & 16-weeks LD & GM6001 + DMSO vehicle injection & Control for GM6001 administration \\
PT $(n=4)$ & 14-weeks SD & None & Control for recrudesced ovaries \\
PTV $(n=7)$ & 2-weeks LD & DMSO vehicle injection & Control for inhibitor administration \\
PTG $(n=9)$ & 14-weeks SD & & Experimental group \\
& 2-weeks LD & GM6001+DMSO vehicle injection & \\
\hline
\end{tabular}


$60 \mu \mathrm{m}$ of tissue and mounted onto adhesion slides. Slides containing six sections per animal $(\geq 180 \mu \mathrm{m}$ between each section) were then deparaffinized in xylene, rehydrated through a graded series of ethanol solutions, and stained with hematoxylin and eosin. Ovarian structures were then counted according to the following groups: preantral follicles (with oocytes), antral follicles (with oocytes), atretic follicles, $\mathrm{CL}$, and eosinophilic terminal atretic follicles. Counts from all sections were evaluated and unique structures were counted for each ovary and therefore each animal.

\section{Gelatin zymography}

MMP2 and MMP9 activity was assessed by gelatin zymography as described before (Shahed \& Young 2008). In short, frozen uterine tissues were homogenized (1:5) in $0.1 \mathrm{M}$ Tris- $\mathrm{HCl}$ ( $\mathrm{pH} 7.6)$ buffer containing $5 \mathrm{mM} \mathrm{CaCl}_{2}, 150 \mathrm{mM} \mathrm{NaCl}, 0.05 \%$ Brij 35, $0.02 \% \mathrm{NaN}_{3}, 1 \%$ Triton X-100, and protease inhibitor cocktail and centrifuged for $30 \mathrm{~min}$ at $3750 \mathrm{~g}$ at $4{ }^{\circ} \mathrm{C}$. Total protein (mg/uterus weight) was determined by the Bradford method (Bio-Rad). Gelatin zymography was performed using precast zymography gels according to manufacturer's directions (Bio-Rad). An aliquot containing $40 \mu \mathrm{g}$ total protein/lane was applied on the gel. Electrophoresis was conducted at constant voltage $(100 \mathrm{~V})$ for $90 \mathrm{~min}$. Gels were then washed in two changes (30 min each) of renaturation buffer $(2.5 \%$ Triton $\mathrm{X}-100)$ and subsequently incubated overnight at $37^{\circ} \mathrm{C}$ in zymogram development buffer $(50 \mathrm{mM}$ Tris, $200 \mathrm{mM} \mathrm{NaCl}$, $5 \mathrm{mM} \mathrm{CaCl}_{2}$, and $0.02 \%$ Brij pH 7.5; Bio-Rad). Gels were stained in $0.5 \%$ Coomassie blue R250, destained until bands were visible, and photographed. Band density was determined using NIH image software as directed.

\section{MMP immunohistochemistry}

Sectioned ovary tissue $(n=4-9$ arbitrarily determined females from the PTV and PTG groups) was stained using MMP1, MMP2, and MMP3 antibodies (collagenase, gelatinase, and stromelysin). Primary antibody (Chemicon, Temecula, CA, USA) was applied to the sections in an empirically determined dilution (1:200 for MMP1 and MMP2, 1:400 for MMP3). Biotinylated goat anti-rabbit (MMP1) or horse anti-mouse (MMP2, MMP3) IgG (Vectastain Elite ABC kit; Vector Laboratories, Burlingame, CA, USA) and Vector NovaRed Substrate kit (Vector Laboratories) were used to visualize the antigen. Sections processed in the absence of primary antibody were used as negative controls. Characteristic extent of staining was noted for four to six ovarian sections per animal for each MMP ( $\geq 180 \mu \mathrm{m}$ between each section). A semiquantitative analysis of staining extent was conducted and sections were given a numerical value ranging from 0 to 3 . A score of 0 indicated no staining; a score of 1 meant faint staining was apparent in at least some of the cells, a score of 2 indicated medium-intense staining that extended throughout the section across multiple follicles, and a score of 3 specified intense staining throughout follicles and some stroma. Results for individual animals were averaged per group and analyzed.

\section{Real-time Q-PCR of MMP2 and MMP9}

MMP2 and MMP9 mRNA expression was assessed by qPCR. Total RNA was isolated from ovarian tissue with Trizol (Invitrogen) and cDNA was synthesized exactly as described before (Shahed \& Young 2009). Primers were designed using Siberian hamster sequences (PCR products extracted and sequenced as described in Shahed \& Young 2009) and OligoPerfect Designer software (Invitrogen) using the MX3000 cycler manual design criteria (Stratagene Corp., Cedar Creek, TX, USA). Primer sequences used were MMP2 (forward) ATG ATG TCA GCT TCC CCA TC; MMP2 (reverse) ACC TGG ACC CTG AAA CAG TG; MMP9 (forward) ACT TTG GAA ACG CAA ATG GT; MMP9 (reverse) AGT CTC TCA CTG GGG CAG AA. A MX3000 thermocycler was used with Absolute QPCR SYBR green mix (ABgene, Surrey, UK) for Q-PCR. The PCR reaction mix contained $1 \mu \mathrm{l}$ CDNA (1:5 dilution of cDNA transcribed using $1 \mu \mathrm{g}$ total RNA) plus $1 \mu \mathrm{l}$ each of forward and reverse primers ( $80 \mathrm{nM}$ concentration) plus $6 \mu \mathrm{l}$ SYBR green mix plus $4 \mu \mathrm{l}$ water (Promega DNase, RNase, protease free) to a total volume of $12 \mu \mathrm{l}$. PCR cycles consisted of $15 \mathrm{~min}$ hold at $95^{\circ} \mathrm{C}$ ( 1 cycle), then 40 amplification cycles at appropriate $T_{\mathrm{m}}\left(60{ }^{\circ} \mathrm{C}\right.$ for MMP2 and $62{ }^{\circ} \mathrm{C}$ for MMP9), extension $\left(1 \mathrm{~min}\right.$ at $72{ }^{\circ} \mathrm{C}$ ) followed by dissociation. Nontemplate negative controls and standards were included in each PCR analysis, and PCR products were analyzed on agarose gels to confirm bp size and to visualize potential secondary and nonspecific amplification. For the standard curve, cDNAs from all samples were pooled and a four-point curve was included with each run (for gene of interest and housekeeping genes). The relative amounts of mRNA (arbitrary units) were calculated using standard curves of each gene of interest and housekeeping gene HPRT1, and the ratio of gene of interest to HPRT1 was calculated.

\section{Statistical analysis}

All data presented were analyzed using Prism 4 statistical software 240 package (GraphPad Software, Inc., San Diego, CA, USA). One-way ANOVAs were performed on all groups and represented by \pm s.E.M. If results were significant, using $P<0.05$, the Neuman-Keuls post hoc test or the Bonferroni multiple comparison test was used to determine differences among experimental groups. To reduce variance in the plasma $\mathrm{E}_{2}$ concentration analysis, a log transform was used. Owing to the nonparametric nature of the terminal atretic follicle data, the Kruskal-Wallis analysis with Dunn's post hoc multiple comparison test was used for these data.

\section{Declaration of interest}

The authors declare that there is no conflict of interest that could be perceived as prejudicing the impartiality of the research reported.

\section{Funding}

This work was supported by NIH SCORE grants (S06GM063119-05; 1SC3GM089611-01, K A Young), the 
CSULB Provost's Undergraduate Student Summer Stipend Program for Research, Scholarly and Creative Activity (J Whited and C F McMichael), and the Howell/CSUPERB Research Fellowship (C F McMichael).

\section{Acknowledgements}

We thank the CSULB Reproductive Biology Laboratory, particularly Lisa Vrooman, Jamie Simmons, Chloe Matula, Steve Dolan, Hani Ahdab, Mustafa Albassleh, and Kerri Loke for aid in tissue collection and processing.

\section{References}

Bauvois B 2001 Transmembrane proteases in focus: diversity and redundancy? Journal of Leukocyte Biology 70 11-17.

Butler GS, Will H, Atkinson SJ \& Murphy G 1997 Membrane-type-2 matrix metalloproteinase can initiate the processing of progelatinase $\mathrm{A}$ and is regulated by the tissue inhibitors of metalloproteinases. European Journal of Biochemistry 244 653-657. (doi:10.1111/j.1432-1033.1997. t01-1-00653.x)

Curry TE Jr \& Osteen KG 2001 Cyclic changes in the matrix metalloproteinase system in the ovary and uterus. Biology of Reproduction 64 1285-1296. (doi:10.1095/biolreprod64.5.1285)

Curry TE Jr \& Osteen KG 2003 The matrix metalloproteinase system: changes, regulation, and impact throughout the ovarian and uterine reproductive cycle. Endocrine Reviews 24 428-465. (doi:10.1210/er. 2002-0005)

Dodge JC, Kristal MB \& Badura LL 2002 Male-induced estrus synchronization in the female Siberian hamster (Phodopus sungorus sungorus). Physiology and Behavior 77 227-231. (doi:10.1016/S0031-9384(02) 00851-X)

Evaul K \& Hammes SR 2008 Cross-talk between G protein-coupled and epidermal growth factor receptors regulates gonadotropin-mediated steroidogenesis in Leydig cells. Journal of Biological Chemistry 283 27525-27533. (doi:10.1074/jbc.M803867200)

Fata JE, Ho AT-V, Leco KJ, Moorehead RA \& Khokha R 2000 Cellular turnover and extracellular matrix remodeling in female reproductive tissues: functions of metalloproteinases and their inhibitors. Cellular and Molecular Life Sciences 57 77-95. (doi:10.1007/s000180050500)

Galardy RE, Cassabonne ME, Giese C, Gilbert JH, Lapierre F, Lopez H, Schaefer ME, Stack R, Sullivan M, Summers B et al. 1994 Low molecular weight inhibitors in corneal ulceration. Annals of the New York Academy of Sciences 732 315-323. (doi:10.1111/j.1749-6632.1994.tb24746.x)

Grobelny D, Poncz L \& Galardy RE 1992 Inhibition of human skin fibroblast collagenase, thermolysin, and Pseudomonas aeruginosa elastase by peptide hydroxamic acids. Biochemistry 31 7152-7154. (doi:10.1021/ bi00146a017)

van den Hurk R, Dijkstra G \& de Jong DH 2002 Enhanced serum oestrogen levels and highly steroidogenic, luteinized atretic follicles in the ovaries of the Djungarian hamster (Phodopus sungorus) kept under a short photoperiod from birth. European Journal of Endocrinology 147 701-710. (doi:10.1530/eje.0.1470701)

Jo M, Thomas LE, Wheeler SE \& Curry TE Jr 2004 Membrane type 1-matrix metalloproteinase (MMP)-associated MMP-2 activation increases in the rat ovary in response to an ovulatory dose of human chorionic gonadotropin. Biology of Reproduction 70 1024-1032. (doi:10.1095/ biolreprod.103.023499)

Kabithe EW \& Place NJ 2008 Photoperiod-dependent modulation of antiMüllerian hormone in female Siberian hamsters, Phodopus sungorus. Reproduction 135 335-342. (doi:10.1530/REP-07-0423)

Kanekura T, Chen X \& Kanzaki T 2002 Basigin (CD147) is expressed on melanoma cells and induces tumor cell invasion by stimulating production of matrix metalloproteinases by fibroblasts. International Journal of Cancer 99 520-528. (doi:10.1002/ijc.10390)
Kessenbrock K, Plaks V \& Werb Z 2010 Matrix metalloproteinases: regulators of the tumor microenvironment. Cell 141 52-67. (doi:10. 1016/j.cell.2010.03.015)

Leib SL, Leppert D, Clements J \& Täuber MG 2000 Matrix metalloproteinases contribute to brain damage in experimental pneumococcal meningitis. Infection and Immunity 68 615-620. (doi:10.1128/IAI.68. 2.615-620.2000)

Liu K, Wahlberg P, Leonardsson G, Hagglund A-C, Ny A, Boden I, Wibom C, Lund LR \& Ny T 2006 Successful ovulation in plasminogendeficient mice treated with the broad-spectrum matrix metalloproteinase inhibitor galardin. Developmental Biology 295 615-622. (doi:10.1016/j. ydbio.2006.03.046)

Moffatt-Blue CS, Sury JJ \& Young KA 2006 Short photoperiod-induced ovarian regression is mediated by apoptosis in Siberian hamsters (Phodopus sungorus). Reproduction 131 771-782. (doi:10.1530/rep.1. 00870)

Nothnick WB 2003 Tissue inhibitor of metalloproteinase-1 (TIMP-1) deficient mice display reduced serum progesterone levels during corpus luteum development. Endocrinology 144 5-8. (doi:10.1210/en.2002220963)

Salverson TJ, McMichael GE, Sury JJ, Shahed A \& Young KA 2008 Differential expression of matrix metalloproteinases during stimulated ovarian recrudescence in Siberian hamsters (Phodopus sungorus). General and Comparative Endocrinology 155 749-761. (doi:10.1016/ j.ygcen.2007.09.003)

Schlatt S, Niklowitz P, Hoffmann K \& Nieschlag E 1993 Influence of short photoperiods on reproductive organs and estrous cycles of normal and pinealectomized female Djungarian hamsters, Phodopus sungorus. Biology of Reproduction 49 243-250. (doi:10.1095/biolreprod49.2.243)

Shahed A \& Young KA 2008 Differential activity of matrix metalloproteinases (MMPs) during photoperiod induced uterine regression and recrudescence in Siberian hamsters (Phodopus sungorus). Molecular Reproduction and Development 75 1433-1440. (doi:10.1002/mrd. 20879)

Shahed A \& Young KA 2009 Differential ovarian expression of KiSS-1 and GPR54 during the estrous cycle and photoperiod induced recrudescence in Siberian hamsters (Phodopus sungorus). Molecular Reproduction and Development 76 444-452. (doi:10.1002/mrd.20972)

Shofuda K, Yasumitsu H, Nishihashi A, Miki K \& Miyazaki K 1997 Expression of three membrane-type matrix metalloproteinases (MT-MMPs) in rat vascular smooth muscle cells and characterization of MT3-MMPs with and without transmembrane domain. Journal of Biological Chemistry 272 9749-9754. (doi:10.1074/jbc.272.15.9749)

Smith MF, Gutierrez CG, Ricke WA, Armstrong DG \& Webb R 2005 Production of matrix metalloproteinases by cultured bovine theca and granulosa cells. Reproduction 129 75-87. (doi:10.1530/rep.1.00381)

Sun I \& Hemler ME 2001 Regulation of MMP-1 and MMP-2 production through CD147/extracellular matrix metalloproteinase inducer interactions. Cancer Research $612276-2281$.

Vrooman LA \& Young KA 2010 Ovarian matrix metalloproteinases are differentially regulated during the estrous cycle but not during short photoperiod induced regression in Siberian hamsters (Phodopus sungorus). Reproductive Biology and Endocrinology 8 79. (doi:10. 1186/1477-7827-8-79)

Wahlberg P, Bodén I, Paulsson J, Lund LR, Liu K \& Ny T 2007 Functional corpora lutea are formed in matrix metalloproteinase inhibitor-treated plasminogen-deficient mice. Endocrinology 148 1226-1234. (doi:10. 1210/en.2006-0669)

Young KA \& Stouffer RL 2004 Gonadotropin and steroid regulation of matrix metalloproteinases and their endogenous tissue inhibitors in the developed corpus luteum of the rhesus monkey during the menstrual cycle. Biology of Reproduction 70 244-252. (doi:10.1095/biolreprod. 103.022053)

Received 12 July 2010

First decision 17 August 2010

Revised manuscript received 21 September 2010

Accepted 29 September 2010 